\title{
The effects of hippocampal and cortical lesions on single-alternation go, no-go acquisition in rats*
}

\author{
DON W. WALKER + V.A. Hospital, Gainesville, Fla. 32601 \\ and \\ LARRY W. MEANS $\uparrow$ and ROBERT L. ISAACSON \\ University of Florida, Gainesville, Fla. 32601
}

Rats with hippocampal ablations, those with cortical control lesions, and normals were trained on a go/no-go single-alternation task in an operant situation. Hippocampectomized animals were facilitated, compared to normals, in acquisition in terms of latency measure (latency to first press on go trials/latency to first press on no-go trials). Cortically ablated animals were deficient compared to normals, in terms of a response measure (total responses on no-go trials/total responses on go trials). An $\mathrm{S}^{\mathrm{D}}$ was added to go trials after the animals attained a low-level criterion and was eliminated after 10 sessions. As measured by performance tests given after elimination of the $S^{D}$, no group differences were found in utilization of the $S^{D}$.

Recently it has been demonstrated that bilateral hippocampectomy in rats results in facilitated acquisition of a single-alternation go/no-go task (Means, Walker. \& Isaacson, in press). A similar observation has been made by Brown, Marco, \& Kaufman (1969) using cats as Ss. The rat study involved a discrete-trial operant situation. Each trial consisted of turning on the houselight and presenting a retractable lever for $20 \mathrm{sec}$ and was followed by a 10-sec ITI, during which the houselight was turned off and the lever retracted. Barpresses were reinforced on alternate trials. The hippocampectomized rats were facilitated, compared to the normal animals, as determined by ratios based on the latency to first press and total number of presses on go and no-go trials, while the cortical control animals showed a significant deficit on the response measure.

From observation of the animals, it was speculated that the observed facilitation resulted from the hippocampectomized rats' attending more to the relevant cues and less to the irrelevant cues early in training than did the normal and cortical control animals (Means et al, in press). After only a couple of sessions, the hippocampally damaged animals began to spend most of their time around the lever or food cup, while the control animals continued to explore the entire box.

The present study was an attempt both to replicate the hippocampal facilitation and cortical deficit and to test the attention hypothesis concerning the facilitation. Therefore, hippocampally aspirated, cortically lesioned, and nomal

*Supported by Grants MH-12574, MH-10320. MS-16384-01, and the Veterans Administration.

$\div$ Postdoctoral fellows during this investigation at the Center for Neurobiological Sciences. University of Florida, Gainesville. Florida. animals were trained to a low-level performance criterion on the go/no-go alternation task. Upon reaching the criterion, an $\mathrm{S}^{\mathrm{D}}$ was introduced. A light over the lever was illuminated on all go or reinforced trials. Subsequently the animals were tested without the $\mathrm{S}^{\mathrm{D}}$. It was reasoned that early in training the hippocampectomized animals would begin to attend to whatever cues they used to perform the alternation and ignore the light over the bar, while the control animals would attend to and utilize the light to perform the discrimination. Thus, we predicted that subsequent removal of the light would not affect the hippocampally damaged animals but would disrupt both the cortically damaged and normal animals.

\section{SUBJECTS}

Twelve male hooded rats, approximately 120 days old at the time of surgery, were employed as Ss. They were housed individually and were maintained on a $12-\mathrm{h}$ light, 12-h dark cycle.

\section{SURGERY AND}

HISTOLOGICAL PROCEDURES

Four rats received bilateral aspirations of the hippocampus and overlying cortex, four received control lesions involving only the overlying cortex, and four served as unoperated controls. Upon completion of behavioral testing, the lesioned rats were sacrificed and perfused with saline and formalin. The brains were removed, embedded in celloiden, sectioned at $30 \mu$, and every 10th section was stained with thionin. Reconstructions of the largest and smallest lesion for each group are shown in Fig. 1.

\section{APPARATUS}

All behavioral testing was done in a modified Lehigh Valley test chamber (No. 1417). The test chamber had a retractable lever at one end of the box and a pellet magazine at the opposite end. The animals had to traverse the length of the box, 12 in., to obtain pellets following reinforced barpresses.

\section{PROCEDURE}

Following 14 days recuperation, all Ss were given two sessions of magazine training followed by daily 10-min FI 2-sec sessions until they accumulated 275 reinforced responses. Throughout the study, Ss were maintained at $85 \%$ of their ad lib body weight and reinforced with .045-g Noyes pellets.

The single-alternation go/no-go training was initiated on the day following completion of the FI-2 training. Each animal received 20 trials per day. Each trial consisted of a 20-sec presentation of a retractable lever and was followed by a $10-\sec$ ITI, during which the lever was retracted. On the odd-numbered trials the animals were reinforced for pressing on a FI 2 -sec schedule, and on the even-numbered trials the rats were not reinforced. The dependent variables were the latency to the first press and the number of presses on each trial. Also, the number of reinforced presses was recorded. Each animal was trained until the ratio of the latency to the first response on go trials to no-go trials (latency-ratio) and the ratio of the number of responses on no-go trials to go trials (response ratio) were both less than 1.00 for 2 consecutive days or for 30 sessions, whichever occurred first. When an animal had reached the ratio criterion or received 30 sessions, an $S^{D}$ was added on
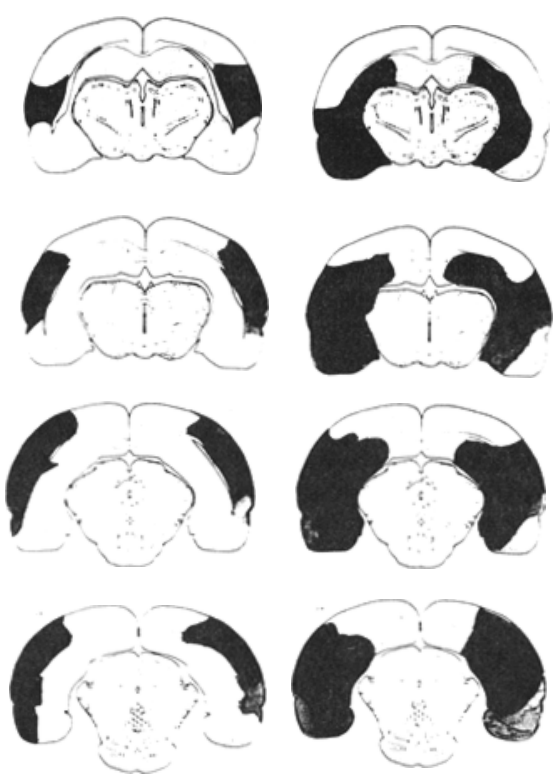

Fig. 1. Reconstructions of the smallest and largest lesions for the cortical (left) and hippocampal (right) groups in equally spaced anterior to posterior sections. 


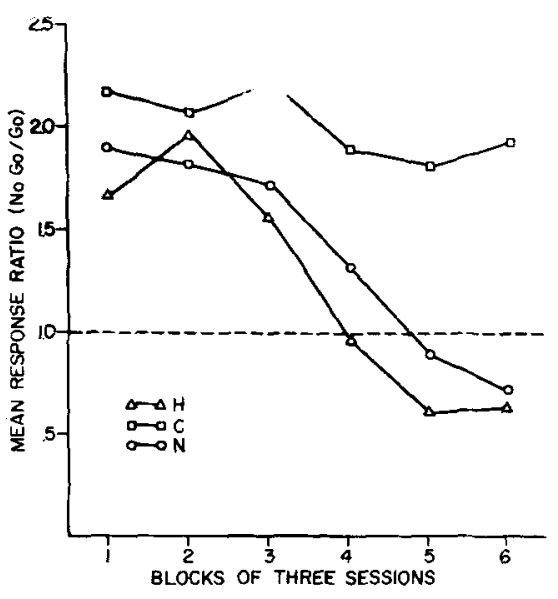

Fig. 2. Mean ratio of total responses on no-go trials to total responses on go trials for each group.

the go trials. The $S^{D}$ consisted of the illumination of a cue light above the bar on all reinforced trials. Each animal received 10 sessions with the $S^{D}$ present on all reinforced trials followed by three test sessions with the $\mathrm{S}^{\mathrm{D}}$ removed.

\section{RESULTS}

Acquisition

Figure 2 shows the mean response ratios number of barpresses on no-go trials/number of barpresses on go trials) for the first 18 acquisition sessions plotted in blocks of three sessions. Note that the hippocampectomized group (Group $\mathrm{H})$ and the normal group (Group N) show consistent improvement over sessions, while the cortically-ablated group (Group C) shows relatively little improvement. An analysis of variance performed on the sum of the response ratios of the first 18 sessions resulted in a significant lesion group effect $(p<.05)$. Subsequent Newman-Keuls tests revealed that the Group $C$ ratio was significantly greater $(p<.05)$ than that of Group $\mathrm{H}$ and Group N, which do not differ significantly

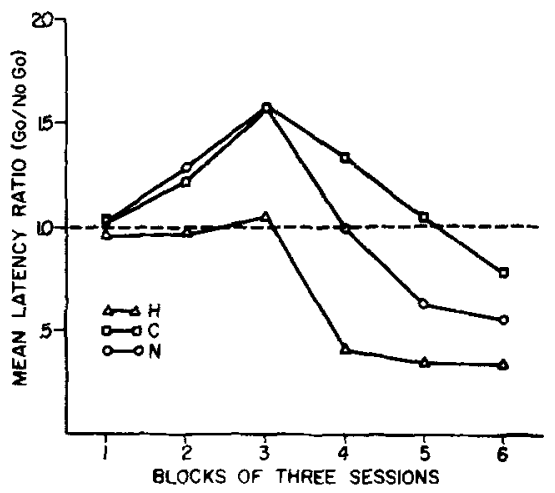

Fig. 3. Mean ratio of the latency to the first response on go trials to the latency of the first response on no-go trials for each group. from one another. Thus, $G$ oup $C$ showed a significant deficit relative to the other two groups with respect to response ratio. Examination of the absolute number of go and no-go responses across sessions revealed that the present data are almost identical to that of the earlier study (Means et al, in press). During the first few sessions all groups increased responding on both go and no-go trials. This was followed by a rapid decrease in responding on the no-go trials by the hippocampal group. Several sessions later, the normal group showed a less dramatic decrease in no-go responses, while the corticals continued to respond approximately twice as often on no-go trials as on go trials throughout the first 18 sessions.

Latency ratios (latency to first barpress on go trials/latency to first barpress on no-go trials) for the three groups on the first 18 sessions are shown in Fig. 3. Note that Group H had a lower latency ratio than both of the other groups throughout. An analysis of variance performed on the sum of the latency ratios for the first 18 sessions produced a significant lesion group effect $(p<.01)$. Subsequent Newman-Keuls tests revealed that Group $\mathrm{H}$ outperformed both Group N $(p<.01)$ and Group C $(p<.01)$. Group $N$ did not differ reliably from Group C. Therefore, Group H showed a significant facilitation on acquisition relative to the other two groups as measured by the latency ratio. Examination of the absolute latencies on go and no-go trials across sessions revealed that the present data are almost identical to our previous experiment. Latencies on both go and no-go trials decreased for all groups during the first few sessions until go trial latencies reached an asymptote. At this point, no-go latencies began to increase again but increased earlier and more dramatically for the hippocampal group.

To further evaluate the three groups on acquisition, they were compared on the number of sessions required to attain the following performance criteria: response ratio less than 1.00 on 2 consecutive days; latency ratio less than 1.00 on 2 consecutive days; and both ratios less than 1.00 on 2 consecutive days. The criterion results are shown in Table 1 . Group C required significantly more sessions to attain each of the three criteria than did the other two groups $(\mathrm{p}<.01$ in every case), as revealed by analyses of variance followed by Newman-Keuls tests. Group $\mathrm{H}$ did not differ reliably from Group $\mathrm{N}$ on any of the three criterion measures.

In summary, the acquisition analysis revealed that Group $C$ had a sigrificant acquisition deficit relative to the other two groups on both response and latency
Table 1

Mean Number of Sessions to Response, Latency, and Response and Latency Criteria

\begin{tabular}{|c|c|c|c|}
\hline \multirow[b]{2}{*}{ Group } & \multirow[b]{2}{*}{ Response } & \multicolumn{2}{|l|}{ Critcrion } \\
\hline & & Latency & $\begin{array}{c}\text { Response } \\
\text { and } \\
\text { Latency }\end{array}$ \\
\hline Hippocampal & 10.507 & 8.507 & 10.75 \\
\hline Cortical & 28.50 & 13.257 & 28.50 \\
\hline Normal & $14.00 \mathrm{~J}$ & $9.00\rfloor$ & 14.25 \\
\hline
\end{tabular}

Note- "J" bars connect groups which differ from one another significantly $(p<.05)$.

criterion measures and on the response ratio over the first 18 sessions. Further, Group $H$ was shown to be significantly facilitated relative to the other two groups over the first 18 sessions, as measured by the latency ratio.

\section{Utilization of $\mathrm{S}^{\mathrm{D}}$}

Several analyses of variance were performed to evaluate changes in either response or latency measures resulting from either the introduction or subsequent removal of the $S^{D}$. Neither the introduction nor the removal of the $S^{D}$ were shown to differentially affect the response or latency ratios of the three groups. Analyses-of-difference scores based on either the response or latency ratios of the last 2 days of acquisition and the first 2 days of $S D$ training failed to produce significant results. The same analysis performed on difference scores based on the last 2 days of $S^{D}$ training and the first 2 days of testing with no $S^{D}$ also failed to produce any significant finding. Thus, the present study failed to produce any evidence that changes in the available relevant stimuli differentially affected the groups. One observation concerning the $\mathrm{S}^{\mathrm{D}}$ training should be mentioned, however. Two cortically ablated animals failed to reach the low-level acquisition criterion in the allowed 30 sessions. These two animals began to show significant improvement only after the $S^{D}$ was introduced, while rats in the other groups showed relatively more improvement during the initial acquisition without the $S^{D}$.

\section{DISCUSSION}

Hippocampectomy was found to facilitate acquisition of go/no-go, as measured by latency to the first response on each trial (latency ratio). This result confirms our previous observation of facilitation in the acquisition of go/no-go following hippocampal lesions (Means et al, in press). On the other hand, no reliable differences between Group $\mathrm{H}$ and Group $\mathrm{N}$ were found, as measured by number of responses on each trial (response ratio) or number of sessions to each criterion, although Group $\mathrm{H}$ was superior on all 
measures. The failure to demonstrate facilitation in Group H on the response ratio and criteria measures is most likely explained by the small group size. analysis of response ratios over only 18 sessions in the present study, as compared to 33 sessions in the original study, and the necessarily low performance-level criteria used.

The severe cortical deficit, as measured by response ratios and criteria measures, and the absence of a cortical deficit (compared to Group N), as measured by latency ratios, also confirm the results of our previous study. It should be pointed out that although the hippocampal group had essentially the same cortex removed as the cortical group, facilitation was still observed. Hippocampectomy either eliminated or compensated for the functional disruption produced by cortical destruction. During evaluation of the histology, it was noticed that the cortical destruction in the animals in the present experiment was more lateral than in the previous study. An investigation is under way, attempting to define the location and extent of cortical destruction involved in producing the observed deficit in go/no-go acquisition.

The present experiment provided no clear evidence that hippocampectomized rats attended to the redundant $S \mathrm{D}$ less than did cortically ablated or normal rats, as measured by performance changes following $S^{D}$ removal. However, the $S^{D}$ may have been introduced too soon (before alternation cues began to be utilized by any group) or too late (after all groups had begun to utilize alternation cues) for an adequate test of the attention hypothesis that was tentatively proposed. It should be noted, however, that two of the rats in the cortical group only began to show improvement after the $\mathrm{SD}^{\mathrm{D}}$ was introduced. Thus, the authors feel that the attention hypothesis stated above cannot be dismissed on the basis of the present study.

The previous (Brown et al, 1969; Means et al, in press) and present results are inconsistent with either a memory hypothesis (see Milner, 1968) or response inhibition hypothesis (see Douglas, 1967 and Kimble, 1968) of hippocampal function. The hippocampal group was able to inhibit responses on nonreinforced no-go trials as well (response ratio) as or better (latency ratio) than normal animals. The hippocampus, thus, apparently is not necessary for response inhibition in this task, nor does hippocampectomy produce a response inhibition deficit. On the other hand, the severe cortical deficit in the ability to withhold responses on no-go trials found in the present and previous (Means et al, in press) experiments suggest that the postereo-lateral cortex overlying the hippocampus does subserve a response inhibition function.

\section{REFERENCES}

BROWN, T. S., KAUFMAN, P. G., \& MARCO, L. A. The hippocampus and response perseveration in the cat. Brain Research, 1969 , $12,86-88$.

DOUGLAS, R. J. The hippocampus and behavior. Psychological Bulletin, 1967, 67, 416-442.

KIMBLE, D. P. The hippocampus and internal inhibition. Psychological Bulletin, 1968, 70 , 285-295.

MEANS, L. W., WALKER, D. W., \& ISAACSON. R. L. lacilitated single alternation go, no-go performance following hippocampectomy in the rat. Journal of Comparative \& Physiological Psychology, in press.

MILNER, B. Disorders of memory after brain lesions in man. Neuropsychologia, 1968, 6, 175-179.

\section{Flavor preferences in laboratory rats*}

\author{
FRED P. VALLE \\ University of British Columbia, Vancouver 8 , Canada
}

One group of rats (Group A-B) was reared on an almond-flavored diet, a second group (Group B-A) on a banana-flavored diet, and a third group (Group C-C), on an unflavored diet. The groups were then given both the banana and almond diets for 2 days. Results showed all three groups preferred almond to banana (almond preference), with Group B-A showing the strongest preference (novelty preference). Results of a second set of tests, following upon an interval during which Groups A-B and B-A had their maintenance flavors switched, were generally consistent with the above but also indicated that a diet's preferability may depend upon an interaction between its primacy, its familiarity, and its attractiveness.

Bronson (1966) reports that laboratory rats that have been fed one type of food

\footnotetext{
*This research was supported by the National Research Council of Canada. Sara Mitchell assisted in collecting the data.
}

from infancy fail to show a preference for that food when given a choice between it and a novel diet. In fact, Bronson's Ss showed an initial preference for the novel diet. Welker \& King (1962) also report that laboratory rats prefer the more novel of two standard types of laboratory chow [see Galef (1970) for information concerning feral rats].

The present study was undertaken partly to develop a procedure for producing a multiflavored diet and partly to replicate the findings of the earlier studies, with the addition of a "control" group for which each of the two test diets was novel.

\section{SUBJECTS}

The Ss were 31 Long-Evans-derived rats bred in the psychology colony at the University of British Columbia.

\section{PROCEDURE}

On the basis of pilot observations, the following procedure was developed for preparing flavored diets. Purina rat pellets were ground into powder and $500 \mathrm{~g}$ of this powder was mixed with $100 \mathrm{ml}$ of Mazola oil. This produced a slightly cohesive food mixture that was resistant to spillage. Three different flavors of food were then prepared by (1) leaving the food mixture unadulterated or (2) adding $5 \mathrm{ml}$ of either banana food flavoring or almond food flavoring to $40 \mathrm{~g}$ of the food mix ture.

As soon as it was determined that three female rats were pregnant, each female was placed on one of the three diets. Hence, one female, whose litter of seven is hereafter referred to as Group C-C, was fed the basic unflavored food mixture. A second female, whose litter of 12 comprised Group A-B, was put on the almond-flavored diet. The third female, whose littter of 12 comprised Group B-A, was placed on the banana-flavored diet.

The females and pups were maintained on these diets following parturition and thoughout the rearing period. Following weaning at 21 days, when they were placed in individual cages, the pups were maintained on the diets for an additional 15 days. All Ss were then given both the banana and almond diets ( $40 \mathrm{~g}$ of each) for 2 consecutive days, with a daily record kept of the amount of each diet $S$ consumed. These 2 days are referred to as Tests 1 and 2.

Following these tests, the Group C-C Ss were again maintained on the unflavored diet. The Group A-B Ss were switched from the almond diet to the banana diet. And the Group B-A Ss were switched from the banana diet to the almond diet. These conditions were continued for 15 days following which another 2-day choice period (Tests 3 and 4) was given.

\section{RESULTS}

The results are expressed as preference ratios of the form $A /(A+B)$, where $A$ refers to the grams of almond-flavored food consumed on the test day and $B$ refers to the grams of banana-flavored food consumed. The mean preference ratios for 